\title{
Spin rotation formalism for spin tracking
}

\author{
A.U. Luccio
}

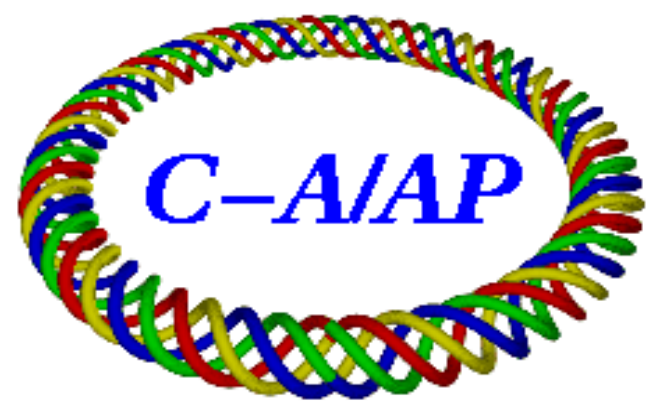

Collider-Accelerator Department Brookhaven National Laboratory

Upton, NY 11973

Notice: This document has been authorized by employees of Brookhaven Science Associates, LLC under Contract No. DE-AC02-98CH10886 with the U.S. Department of Energy. The United States Government retains a non-exclusive, paidup, irrevocable, world-wide license to publish or reproduce the published form of this document, or allow others to do so, for United States Government purposes. 


\title{
Spin rotation formalism for spin tracking *
}

\author{
A.U. Luccio, \\ Brookhaven National Laboratory, Upton, NY
}

February 1, 2008

\begin{abstract}
The problem of which coefficients are adequate to correctly represent the spin rotation in vector spin tracking for polarized proton and deuteron beams in synchrotrons is here re-examined in the light of recent discussions. The main aim of this note is to show where some previous erroneous results originated and how to code spin rotation in a tracking code. Some analysis of a recent experiment is presented that confirm the correctness of the assumptions.
\end{abstract}

\section{Foreword}

This tech. note is an amended version of a previous one, aimed at correcting conceptual errors and other bugs in the same. A few came to my attention by carefully re-reading ("My God, what did I write!"), others were pointed out by colleagues, notably Waldo MacKay and Sateesh Mane, whom I thank. I still believe that many of the arguments proposed may be useful, so I left them in, even if they may be arguable.

\section{The BMT equation}

Spin tracking in a polarized particle accelerator is done in Spink[1] by moving the beam through transport maps created by $M A D$ and rotating the spin at each element by means of $3 \times 3$ matrices. This is the case of vector polarization of, typically, protons or deuterons. For us the spin vector $\mathbf{S}$ is a real, unitary, 3-component vector.

Spin matrices are calculated starting from the BMT equation in the laboratory frame, with no electric field present[2]

$$
\left\{\begin{array}{c}
\frac{d \mathbf{S}}{d t}=\mathbf{S} \times \mathbf{F} \\
\mathbf{F}=\frac{q}{m \gamma}\left[(1+G) \mathbf{B}_{\|}+(1+G \gamma) \mathbf{B}_{\perp}\right]
\end{array}\right.
$$

where

$$
\mathbf{B}_{\|}=(\mathbf{u} \cdot \mathbf{B}) \mathbf{u}
$$

is the component of the local magnetic field in each element of the machine along the trajectory of the particle, and

$$
\mathbf{B}_{\perp}=(\mathbf{u} \times \mathbf{B}) \times \mathbf{u}
$$

is the component of the local magnetic field perpendicular to the trajectory. $\mathbf{u}=\mathbf{v} / \beta c$ is the (normalized) velocity of the particle. $\mathbf{S}$ is in the fixed laboratory coordinates $\hat{\mathbf{X}}=(\hat{x}, \hat{y}, \hat{z})$ and $\mathbf{B}=\mathbf{B}_{\|}+\mathbf{B}_{\perp}$ is the total magnetic field, that in a machine element will be calculated also in $\hat{\mathbf{X}}$. Spink uses the "accelerator convention": $\hat{x}$, radial, $\hat{y}$, vertical and $\hat{z}$, longitudinal. We call $s$ the length of arc along the trajectory.

\footnotetext{
${ }^{*}$ Work performed under the auspices of the U.S.Department of Energy.
} 
Eq.(1) suggests that the coefficient for spin rotation in a field parallel to the trajectory should proportional to $1+G$ and that the coefficient for a field perpendicular to the trajectory should be proportional to $1+G \gamma$. We will see that this is NOT the case.

Many Authors, as S.Y.Lee in his classical book[3], start from Eq.(1) and from the Lorentz equation of motion, to embark in a series of approximations and changes of coordinates, from the laboratory to the moving Frénet-Serret frame and back, to arrive at formulas useful to calculate spin rotation. This derivation led to a coefficient proportional to $1+G \gamma$ for the rotation of the spin due to field components transverse to the trajectory and to $G$ for the rotation due to the longitudinal component of the field. However, it has been pointed out by Mane, Shatunov and Yokoya[?] and others, that the correct answer is to make the spin kick due to the transverse component proportional to $G \gamma$, instead that $1+G \gamma$. This has recently become a subject of important discussions, because the interpretation of experimental data and the formalism in spin tracking codes like Spink by Luccio and Depol by Courant and Ruth[4] would depend on it.

Courant, in a recent note[5], concludes that the discrepancy is due to a different interpretation of which spin coordinates (in the laboratory or in a frame moving with the particle) should be considered more "physical" and therefore should be used, e.g. to calculate depolarization. Clearly a question is still: in which reference a polarimeter "sees" and measures the polarization? On the other hand as it has been forcefully stressed by Mane and Forrest ${ }^{1}$, the strength of a spin resonance does not depend on the frame of reference used.

In this note we want to show that a simple reason for the discrepancy, in some sense equivalent to the argument by Courant, was that in older treatments the curvature of the orbit in elements other than bending magnets was not correctly taken, mixing up in fact which one was the local radius of curvature to use.

For us who do Spink computer tracking, it is not necessary to perform any analytical derivation involving approximations in solving at the same time the Lorentz equations for particle trajectory and the BMT equation for spin rotation, since the position and the velocity of the particle are known at each machine element, having been evaluated independently from the spin by tracking of particle trajectories through an array of transport maps like $6 \times 6 M A D[6]$ supplied matrices.

To save time in multi-turn tracking in a real dynamically changing accelerator lattice we have chosen in Spink to calculate spin rotation using thin element spin kick $3 \times 3$ matrices, with all the limitations and shortcomings that this approach entails. We place these thin matrices in the middle of each "thick" machine element.

By trajectory tracking, at each point in the machine we know the position and the velocity components of a particle in the $\hat{\mathbf{X}}$ laboratory reference

$$
\mathbf{r}=\left(\begin{array}{l}
x \\
y \\
z
\end{array}\right), \quad \mathbf{u}=\left(\begin{array}{c}
u_{x}=x^{\prime} \\
u_{y}=y^{\prime} \\
u_{z}=\left(1-\left(u_{x}^{2}+u_{y}^{2}\right)\right)^{1 / 2} .
\end{array}\right),
$$

The transverse position is taken with respect to a reference or "design" accelerator orbit, which in a synchrotron is straight through some accelerator elements, like quadrupoles, and is curved in bending magnets.

In machine elements with a field transverse to the trajectory the orbit is bent by an angle $\theta$ over an arc; in elements with only longitudinal field, like solenoids, the orbit is not bent. Let us refer to the sketch of Fig.1. The particle coordinates at the entrance are denoted by the superscript $(A)$

$$
\mathbf{r}^{(A)}=(x, y, z)^{(A)}, \mathbf{u}^{(A)}=\left(u_{x}, u_{y}, u_{z}\right)^{(A)},
$$

similarly, in the middle by the superscript $(B)$, and at the exit by $(C)^{2}$. In Spink we average

$$
\mathbf{r}^{(B)}=\frac{\mathbf{r}^{(A)}+\mathbf{r}^{(C)}}{2}, \quad \mathbf{u}^{(B)}=\frac{\mathbf{u}^{(A)}+\mathbf{u}^{(C)}}{2} .
$$

\footnotetext{
${ }^{1}$ S.Mane, E.Forrest, "private communication"

${ }^{2}$ Still, the trajectory tracking is performed in the phase space of the six variables $x, p_{x}, y, p_{y}, \Delta \phi, \Delta E / p c$
} 


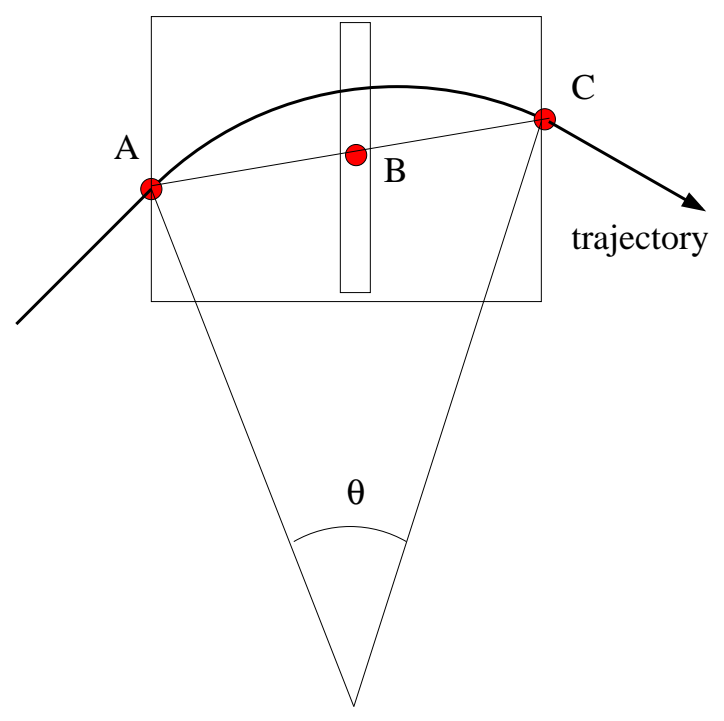

Figure 1: A Transverse field thin spin kick element in the middle of a thick orbit transfer element

$\mathbf{r}^{(B)}$ is the location of the thin spin rotation element, where the magnetic field is calculated in the laboratory coordinates

$$
\mathbf{B}=\left(B_{x}, B_{y}, B_{z}\right)^{(B)} .
$$

\section{The spin rotation matrix for a thin element}

The spin kick is calculated starting from the differential Eq.(1) converted to a finite difference equation

$$
\mathbf{S}^{(C)}=\mathbf{S}^{(A)}+\mathbf{F}^{(B)} \times \mathbf{S}^{(A)} \delta t,
$$

or

$$
\mathbf{S}^{(B+)}=\mathcal{M} \mathbf{S}^{(B+)}
$$

Explicitly ${ }^{3}$

$$
\left(\begin{array}{c}
S_{x} \\
S_{y} \\
S_{z}
\end{array}\right)^{(B+)}=\left(\begin{array}{ccc}
1 & F_{z} \delta t & -F_{y} \delta t \\
-F_{z} \delta t & 1 & F_{x} \delta t \\
F_{y} \delta t & -F_{x} \delta t & 1
\end{array}\right)^{(B)} \cdot\left(\begin{array}{c}
S_{x} \\
S_{y} \\
S_{z}
\end{array}\right)^{(B-)} .
$$

For a machine element where the arc of trajectory is $\delta s$, we have

$$
\delta t=\frac{\delta s}{\beta c},
$$

To build $\mathcal{M}$, using Eq.(1), we need first to build up $\mathbf{B}_{\|}$and $\mathbf{B}_{\perp}$ from the field components in the lab frame where the field is known. Obtain

$$
\mathbf{B}_{\|}=\mathbf{u} \cdot \mathbf{B}\left(\begin{array}{l}
u_{x} \\
u_{y} \\
u_{z}
\end{array}\right) \text {, with } \mathbf{u} \cdot \mathbf{B}=u_{x} B_{x}+u_{y} B_{y}+u_{z} B_{z}
$$

and

$$
\mathbf{B}_{\perp}=\left(\begin{array}{ccc}
u_{y}^{2}+u_{z}^{2} & -u_{x} u_{y} & -u_{x} u_{z} \\
-u_{x} u_{y} & u_{x}^{2}+u_{z}^{2} & -u_{y} u_{z} \\
-u_{x} u_{z} & -u_{y} u_{z} & u_{x}^{2}+u_{y}^{2}
\end{array}\right) \cdot\left(\begin{array}{c}
B_{x} \\
B_{y} \\
B_{z}
\end{array}\right) .
$$

\footnotetext{
${ }^{3}$ The determinant of the following matrix is 1 only in the limit of $\delta t=0$. This is a dear price we pay to convert a differential equation to finite differences in this simple way. Ia a future note we will show how we can build a spin matrix with a determinant of one.
} 
Using Eqs.(4) and (5) the $\mathbf{F}$ vector in Eq.(1) can be written as

$$
\mathbf{F}=\frac{q}{m \gamma}\left(\begin{array}{ccc}
1+\left(u_{y}^{2}+u_{z}^{2}\right) G \gamma+u_{x}^{2} G & -u_{x} u_{y} G(\gamma-1) & -u_{x} u_{y} G(\gamma-1) \\
-u_{x} u_{y} G(\gamma-1) & 1+\left(u_{x}^{2}+u_{z}^{2}\right) G \gamma+u_{y}^{2} G & -u_{y} u_{z} G(\gamma-1) \\
-u_{x} u_{z} G(\gamma-1) & -u_{y} u_{z} G(\gamma-1) & 1+\left(u_{x}^{2}+u_{y}^{2}\right) G \gamma+u_{z}^{2} G
\end{array}\right) \cdot\left(\begin{array}{c}
B_{x} \\
B_{y} \\
B_{z}
\end{array}\right) .
$$

This expression is exact in $\mathbf{u}$. It only contains the approximations implicit in the thin rotation.

\section{Trajectory angle}

Since it is physically appropriate to refer the spin to the direction of propagation of the beam, for spin transmission through a machine element we must multiply the matrix of Eq.(3) by a rotation matrix, to its right and its left. The complete spin transformation will be then

$$
\mathbf{S}^{(C)}=\mathcal{R}(\theta / 2) \cdot \mathcal{M} \cdot \mathcal{R}(\theta / 2) \mathbf{S}^{(A)},
$$

with $\theta$ the angle of curvature of the beam in the machine element. $\mathcal{M}$ is the $3 \times 3$ spin rotation matrix of Eq.(3), and $\mathcal{R}(\theta / 2)$ is a matrix to rotate the system of coordinates from (A) to (B), and again to rotate from (B) to $(\mathrm{C})$, such that the spin vector at the exit of the element will be referred to the new direction of propagation of the beam.

The angle $\theta$ is usually small in a large synchrotron, but if it is not we should already in $M A D$ longitudinally subdivide the machine element in slices until it is so. To simplify the analysis on this paper, through expansion in powers of $\theta$ we could see that it is, to second order

$$
\mathcal{R}(\theta / 2) \cdot \mathcal{M} \cdot \mathcal{R}(\theta / 2) \approx \mathcal{M} \cdot \mathcal{R}(\theta)
$$

Let us use the system of laboratory coordinates $\hat{\mathbf{X}}=(\hat{(x)}, \hat{y}, \hat{z})$ at $(\mathrm{B})$ where the magnetic field and $\mathcal{M}$ are calculated, and at the exit (C) use a new set of coordinates referred to the particle trajectory $\hat{\mathbf{E}}=\left(\hat{e}_{1}, \hat{e}_{2}, \hat{e_{3}}\right)$ that will defined below.

The general transformation of the coordinates of a vector from coordinates at (B) to coordinates at $(\mathrm{C})$ is

$$
\mathbf{S}^{(C)}=\left(\begin{array}{ccc}
\cos \alpha_{1} & \cos \beta_{1} & \cos \gamma_{1} \\
\cos \alpha_{2} & \cos \beta_{2} & \cos \gamma_{2} \\
\cos \alpha_{3} & \cos \beta_{3} & \cos \gamma_{3}
\end{array}\right) \cdot \mathbf{S}^{(B)}
$$

where the matrix contains the cosines of the angle that each axis of the reference $\hat{\mathbf{E}}$ makes with the corresponding axis of the reference $\hat{\mathbf{X}}$. In the case of interest here, where the magnetic field at (B) is transverse to the particle motion, the arc is all contained in a plane $(\beta)$ perpendicular to $\mathbf{B}$, as seen in Fig.2, so, the appropriate transformation of coordinate will have $\hat{e}_{3}$ directed along the longitudinal velocity $\overrightarrow{u_{z}}$ at $(\mathrm{C})$, making an angle $\theta$ with the longitudinal velocity at (A), a transverse axis $\hat{e}_{2}$ directed as $\mathbf{B}$ at (B), and the other transverse axis $\hat{e}_{1}$ transverse to the previous two. The new axes are defined as

$$
\left\{\begin{array}{c}
\hat{e}_{3}=u_{x} \hat{x}+u_{y} \hat{y}+u_{z} \hat{z}=\cos \phi \sin \theta \hat{x}+\sin \phi \sin \theta \hat{y}+\cos \theta \hat{z} \\
\hat{e}_{2}=-\sin \phi \hat{x}+\cos \phi \hat{y}, \text { parallel to } \mathbf{B} \\
\hat{e}_{1}=\hat{e}_{2} \times \hat{e}_{3}
\end{array},\right.
$$

with $\phi$ the angle of the field with respect to the vertical $\hat{y}$. These rotation matrix of Eq.(9) for an $\operatorname{arc} \theta$ is

$$
\mathcal{R}(\theta)=\left(\begin{array}{ccc}
\cos \phi \cos \theta & -\sin \phi \cos \theta & \sin \theta \\
-\sin \phi & \cos \phi & 0 \\
-\cos \phi \sin \theta & -\sin \phi \sin \theta & \cos \theta
\end{array}\right) .
$$

The above matrix is obtained by convolution of the rotation $\theta$ in an horizontal plane by a rotation of $\phi$ of the same plane around the longidudinal axis $\hat{z}$

$$
\left(\begin{array}{ccc}
\cos \theta & 0 & \sin \theta \\
0 & 1 & 0 \\
-\sin \theta & 0 & \cos \theta
\end{array}\right) \cdot\left(\begin{array}{ccc}
\cos \phi & \sin \phi & 0 \\
-\sin \phi & \cos \phi & 0 \\
0 & 0 & 1
\end{array}\right)
$$




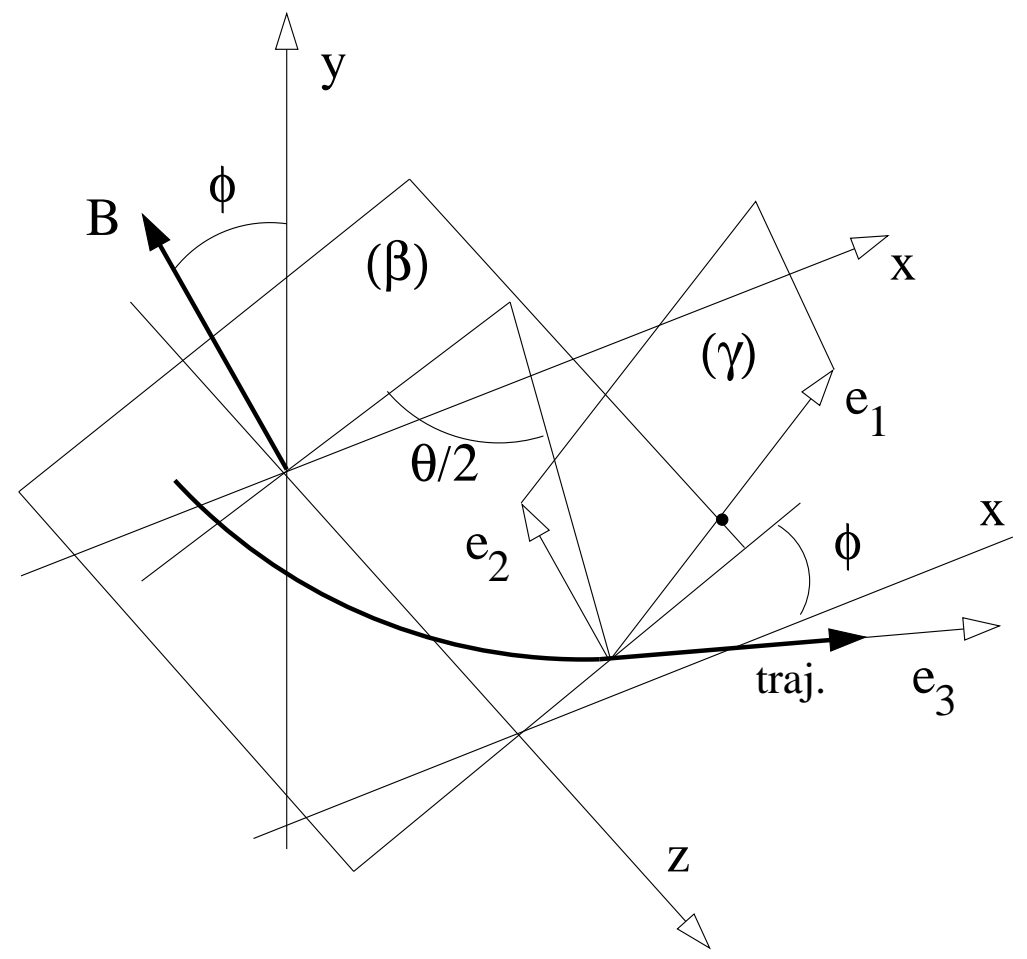

Figure 2: The trajectory of a particle produced by a magnetic field directed along the particle motion is an arc laying in a plane $(\beta)$ perpendicular to $\mathbf{B}$. The plane $(\gamma)$ is normal to the beam velocity.

The arc angle is

$$
\theta=\frac{\int_{A}^{C} \sqrt{\left(x-x^{A}\right)^{2}+\left(y-y^{A}\right)^{2}+\left(y-y^{A}\right)^{2}} d s}{\rho_{\perp}} \approx \frac{\delta s}{\rho_{\perp}},
$$

ratio of the length of arc of trajectory to the radius of curvature in the local field. $\delta s$ is the physical length of the thick machine element. It is also

$$
\frac{1}{\rho_{\perp}}=\frac{q B_{\perp}}{m \gamma \beta c} .
$$

Note that the curvature $1 / \rho_{\perp}$ is not the curvature of the reference orbit, but the curvature of the orbit of the individual particle. In $M A D$ the physical length of a bending magnet is calculated as the length of the arc of trajectory, but the length of a quadrupole is just the length of the hard mass of the device.

The transmission of spin along the accelerator lattice is done by a sequence of spin kicks followed by rotation to coordinates always referred to a plane perpendicular to the velocity, as shown in the cartoon of Fig.3.

Finally, recall that a bending magnet is generally endowed with a uniform vertical magnetic field, that provides a bend always in the same direction for all particles, approximately all by the same angle, while in a quadrupole the field is a function of the actual radial position of the particle, it is transverse, horizontal or vertical, and can be positive or negative. Successive quadrupoles in the lattice provide alternate bends in opposite directions, to keep the beam stable. The physics of spin rotation is otherwise identical for bends or quads and the spin transformation is always completed with a rotation of the type of Eq.(11), albeit with different angles for different particles in the beam. 


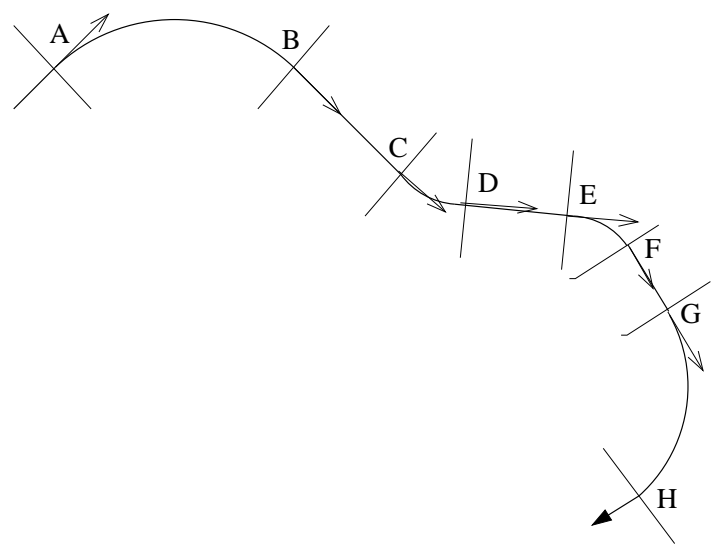

Figure 3: Trajectory of one particle through bends, straights and quadrupoles. The particle reference system for spin has the longitudinal axis constantly directed along the tangent to the trajectory

\section{Examples}

Let us examine three specific examples of spin rotation matrices, showing also how they reduce in approximations respect to the components of the velocity and to $\theta$. Specific cases are:

- Bend: $B_{x}=B_{z}=0, B_{y} \neq 0$.

- Solenoid: $B_{x}=B_{y}=0, B_{z} \neq 0$.

- Quadrupole: $B_{x}, B_{y} \neq 0, B_{z}=0$.

\subsection{Bend}

$$
\mathbf{B}=\left(0, B_{y}, 0\right)
$$

In this case the expression for $\mathbf{F}$ of Eq.(6) reduces to

$$
\mathbf{F}=\frac{q}{m \gamma} B_{y}\left(\begin{array}{c}
-u_{x} u_{y} G(\gamma-1) \\
1+\left(u_{x}^{2}+u_{z}^{2}\right) G \gamma+u_{y}^{2} G \\
-u_{y} u_{z} G(\gamma-1)
\end{array}\right) .
$$

The spin rotation matrix of Eq.(3), including the rotation of coordinates of Eq.(11), becomes

$$
\left(\begin{array}{ccc}
1 & 0 & -F_{y} \delta t \\
0 & 1 & 0 \\
F_{y} \delta t & 0 & 1
\end{array}\right) \cdot\left(\begin{array}{ccc}
\cos \theta & 0 & \sin \theta \\
0 & 1 & 0 \\
-\sin \theta & 0 & \cos \theta
\end{array}\right) .
$$

The dominant component of the velocity vector $\mathbf{u}$ is $u_{z} \approx 1$. To 0 .th order in $u$ it is

$$
\mathbf{F} \approx F_{y} \hat{y} \approx(1+G \gamma) \frac{q B_{y}}{m \gamma} \hat{y}
$$

with $\hat{y}$ the unitary vector pointing along the (vertical) axis. Also, to 0 .th order, for a bend short in length compared to $\rho_{\perp}$, to 1 .st order in $\theta$ it is

$$
\cos \theta=1, \quad \sin \theta=\theta
$$

and because it is

$$
\frac{q}{m \gamma \beta c} B_{y}=\frac{1}{\rho_{\perp}}, \quad \delta t=\frac{\delta s}{\beta c}, \quad \frac{q B_{y} \delta s}{m \gamma \beta c}=\theta
$$


obtain to 0. th order in $\mathbf{u}$ and $\delta s / \rho_{\perp}$

$$
\mathcal{M R}=\left(\begin{array}{ccc}
1 & 0 & -G \gamma \theta \\
0 & 1 & 0 \\
G \gamma \theta & 0 & 1
\end{array}\right)
$$

The rotation matrix got rid of the " 1 " in the $1+G \gamma$ coefficient. To higher order in $u$ and $\theta$ the cancellation of the " 1 " is not complete and for a better approximation we may as well use the complete exact expressions for $\mathcal{M}$ and $\mathcal{R}$.

Since neither $\mathcal{M}$, as stated before, nor $\mathcal{R}$ at this $\theta$ order, have a determinant of 1 , so the matrix of Eq.(12) will have a determinant of 1 only in the limit $\theta \rightarrow 0$. It is

$$
\operatorname{det}[\mathcal{M R}]=1+(G \gamma \theta)^{2},
$$

so, a good parameter for the expansion might be $G \gamma \theta$.

\subsection{Solenoid - no fringe fields}

$$
\mathbf{B}=\left(0,0, B_{z}\right), \quad \theta=0, \quad \mathcal{R}=1
$$

In this case

$$
\mathbf{F}=\frac{q}{m \gamma c} B_{z}\left(\begin{array}{c}
-u_{x} u_{z} G(\gamma-1) \\
-u_{y} u_{z} G(\gamma-1) \\
1+\left(u_{x}^{2}+u_{y}^{2}\right) G \gamma+u_{z}^{2} G
\end{array}\right)
$$

To 0. th order in $u$ it is

$$
\mathbf{F} \approx \hat{z} F_{z} \approx \hat{z} \frac{q}{m \gamma} B_{z} G,
$$

with $\hat{z}$ the unitary vector pointing along the (longitudinal) axis. In this case the spin rotation matrix of Eq.(3) becomes

$$
\left(\begin{array}{ccc}
1 & G \frac{q B_{z}}{m \gamma \beta c} \delta s & 0 \\
-G \frac{q B_{z}}{m \gamma \beta c} \delta s & 1 & 0 \\
0 & 0 & 1
\end{array}\right) .
$$

In a solenoid the coefficient for the spin kick is simply $G$.

\subsection{Quadrupole}

$$
\mathbf{B}=\left(B_{x}, B_{y}, 0\right) .
$$

The formalism is exactly the same as for a bend, only recall that the arc of trajectory lies in a plane perpendicular to B. In extreme case of field only horizontal or vertical it is, respectively

$$
\mathbf{F}=\frac{q}{m \gamma} B_{x}\left(\begin{array}{c}
1+\left(u_{y}^{2}+u_{z}^{2}\right) G \gamma+u_{x}^{2} G \\
-u_{x} u_{y} G(\gamma-1) \\
-u_{x} u_{z} G(\gamma-1)
\end{array}\right) \mathcal{R}\left(\theta_{x}\right), \quad \text { or } \quad \frac{q}{m \gamma} B_{y}\left(\begin{array}{c}
-u_{x} u_{y} G(\gamma-1) \\
1+\left(u_{x}^{2}+u_{z}^{2}\right) G \gamma+u_{y}^{2} G \\
-u_{y} u_{z} G(\gamma-1)
\end{array}\right) \mathcal{R}\left(\theta_{y}\right) .
$$

The spin rotation matrix of Eq.(3) is obtained in the two cases, or in any intermediate case by setting the appropriate value of the angle $\phi$ of the magnetic field with respect to the vertical $\hat{y}$. To 0 .th order in $u$, similarly of done for the bend, we must apply the coordinate rotations of Eq.(11). Like for the bend, as it is immediate to verify, the rotation coefficient becomes proportional to $G \gamma$. The "1" of $1+G \gamma$ is gone for each individual particle in the beam, each with its own $\rho_{\perp}$ and $\theta$.

A final note of caution for an extended quadrupole, at variance with is the case for a bend: since the field is a function of the radial position where the particle is located at each moment, its value is slightly less than in the center of the magnet, so the limits of the thin kick must be "experimentally" tested. 


\subsection{Third order in $\theta$}

As shown before, $1+G \gamma$ becomes $G \gamma$ for transverse field spin kick in each case, in the approximation of a short arc, or small values of $\theta$. The next order is

$$
\cos \theta=1-\frac{\theta^{2}}{2}, \quad \sin \theta=\theta-\frac{\theta^{3}}{6}
$$

In this case we obtain for the rotation matrix, in the case of a bend around $\hat{y}$

$$
\left(\begin{array}{ccc}
1+\left(\frac{1}{2}+G \gamma\right) \theta^{2} & 0 & -G \gamma \theta\left(1-\frac{\theta^{2}}{2}\right)+\frac{2}{3} \theta^{3} \\
0 & 1 & 0 \\
G \gamma \theta\left(1-\frac{\theta^{2}}{2}\right)-\frac{2}{3} \theta^{3} & 0 & 1+\left(\frac{1}{2}+G \gamma\right) \theta^{2}
\end{array}\right),
$$

and similar expression for other orientations.

\section{The polarimeter}

The arguments in the previous section spell out that the correct physical description of the spin is in a reference system that is moving along with the beam, with its longitudinal axis in the direction of the instantaneous longitudinal velcity. The consequences are big in some cases, because the spin kick in an element with transverse field results proprtional to $G \gamma$ and not to $1+G \gamma$. It is surprising that such large effect arises even though spin vector components in the fixed reference and the beam reference are very close to each other, as is seen by the expression of the trasformation of the spin between references, in direction parallel to $\mathbf{u}$

$$
\mathbf{S}_{\|}=(\mathbf{u} \cdot \mathbf{S}) \mathbf{u}
$$

and, in a direction perpendicular to $\mathbf{u}$

$$
\mathbf{S}_{\perp}=(\mathbf{u} \times \mathbf{S}) \times \mathbf{u} .
$$

The explicit expressions are formally identical to Eqs.(4) and (5), i.e.

$$
\mathbf{S}_{\|}=\left(\begin{array}{ccc}
u_{x}^{2} & u_{x} u_{y} & u_{x} u_{z} \\
u_{x} u_{y} & u_{y}^{2} & u_{y} u_{z} \\
u_{x} u_{z} & u_{y} u_{z} & u_{z}^{2}
\end{array}\right) \cdot\left(\begin{array}{c}
S_{x} \\
S_{y} \\
S_{z}
\end{array}\right)
$$

and

$$
\mathbf{S}_{\perp}=\left(\begin{array}{ccc}
u_{y}^{2}+u_{z}^{2} & -u_{x} u_{y} & -u_{x} u_{z} \\
-u_{x} u_{y} & u_{x}^{2}+u_{z}^{2} & -u_{y} u_{z} \\
-u_{x} u_{z} & -u_{y} u_{z} & u_{x}^{2}+u_{y}^{2}
\end{array}\right) \cdot\left(\begin{array}{c}
S_{x} \\
S_{y} \\
S_{z}
\end{array}\right),
$$

where $\mathbf{S}$ is the spin in the fixed frame. To first order in $u$ it is

$$
S_{\|}=S_{z}, \quad S_{\perp, x}=S_{x}, \quad S_{\perp, y}=S_{y},
$$

The polarimeter is attached to the laboratory fixed system of reference. How could it then be sensitive to someting that happens in a reference attached to each particle in the beam? The answer is in the transformation equations we have just written. One can see that the spin representations in both systems are very close, in particular in a straight section of the machine where a polarimeter is normally located, where the value of the transverse components of the velocity, averaged over all particles in the beam, are very close to zero. It would be perhaps interesting to measure the polarization in the proximity of a bending magnet or a quadrupole. 


\section{An experimental verification (with A.Lehrach)}

The key point of the theoretical considerations above as implemented in the code Spink, is contained in the statement in Sec. 2 that follows the description of the behaviour of the spin in a machine element where a magnetic field perpendicular to the trajectory of the particle is present. Previously it was assumed, and also in previous versions of Spink we assumed, that in all machine elements inserted in a straight section and with the direction of the magnetic field transverse to the particle trajectory, the spin kick resulted in first order proportional to $1+G \gamma$, while only in the bends, where all particles make coherently an arc of curve and therefore the reference orbit was curved, the spin kick was to be taken proportional to $G \gamma$, see Subsec. 5.1. Indeed in S.Y.Lee book[3] the formula for a resonance strength, Eq.(3.4), p.97, contains an $1+G \gamma$ coefficient.

In high energy accelerators the difference between $G \gamma$ and $1+G \gamma$ is small, so Spink tracking so far, for RHIC, the AGS, and the main ring of J-Parc, seemed correct and accurately reproduced all measured quantities. A case where the difference would have been clearly seen was the measurements on deuterons on COSY, because the energy was relatively small and moreover the G factor of deuterons is negative. Published results[7] on deuteron spin resonances, induced by a ramping transverse Radio Frequency dipole, whose strength was interpreted using the conventional $1+G \gamma$ show a large discrepancy between measured and calculated values. In this case it was

$$
G \gamma \approx-0.2, \quad 1+G \gamma \approx-0.8
$$

a difference of a factor of 4 !

Tracking with Spink where the RF Dipole kick was made proportional to $G \gamma$ gave a very accurate agreement with the measurements. Fig. 4 shows the comparison between simulation and the experimental curve labeled " $+1+1$ " in Fig. 2 of the cited paper.

In the experiment that we tried to exactly simulate in Spink, the run data were:

$\begin{array}{lll}\text { Deuteron momentum } & 1.858 \mathrm{GeV}, \quad \text { no acceleration } \\ \text { Integrated RF field } B d \ell= & 0.0006 \mathrm{Tesla} \cdot \mathrm{m}\end{array}$ Spin flip frequency $\nu_{m, 0}=\begin{array}{ll}0.919284 \mathrm{MHz}\end{array}$

The expression for the RF Dipole vertical orbit kick used in Spink was

$$
K=\frac{B d \ell}{B \rho} \cos \phi, \quad \phi=2 \pi \int \nu_{m} d t, \quad p_{y}:=p_{y}+K,
$$

with $\nu_{m}$ a tune function that was swept btween the values of $\nu_{m, 0} \pm 150 \mathrm{~Hz}$ in a variable time, interval $\Delta t$ represented on the horizontal axis of the figure. The speed of crossing the resonance was

$$
\alpha=\frac{\Delta \nu_{m}}{\Delta t}[\mathrm{~Hz} / \mathrm{sec}], \quad \Delta f=300 H z .
$$

Note that putting a coefficient $G \gamma$ ONLY in front of the FR Dipole spin kick was a jury-rig. An exact treatment involved a remastering of the Spinl spin rotation algorithm, which was done after this analysis, and the analysis repeated. However, since for this specific example the only meaningful kick was imparted by the RF Dipole through modulation of the vertical orbit via an oscillating horizontal magnetic field, while the effect of the quadrupoles was averaged out to nil, the treatment gave the right result anyway. 


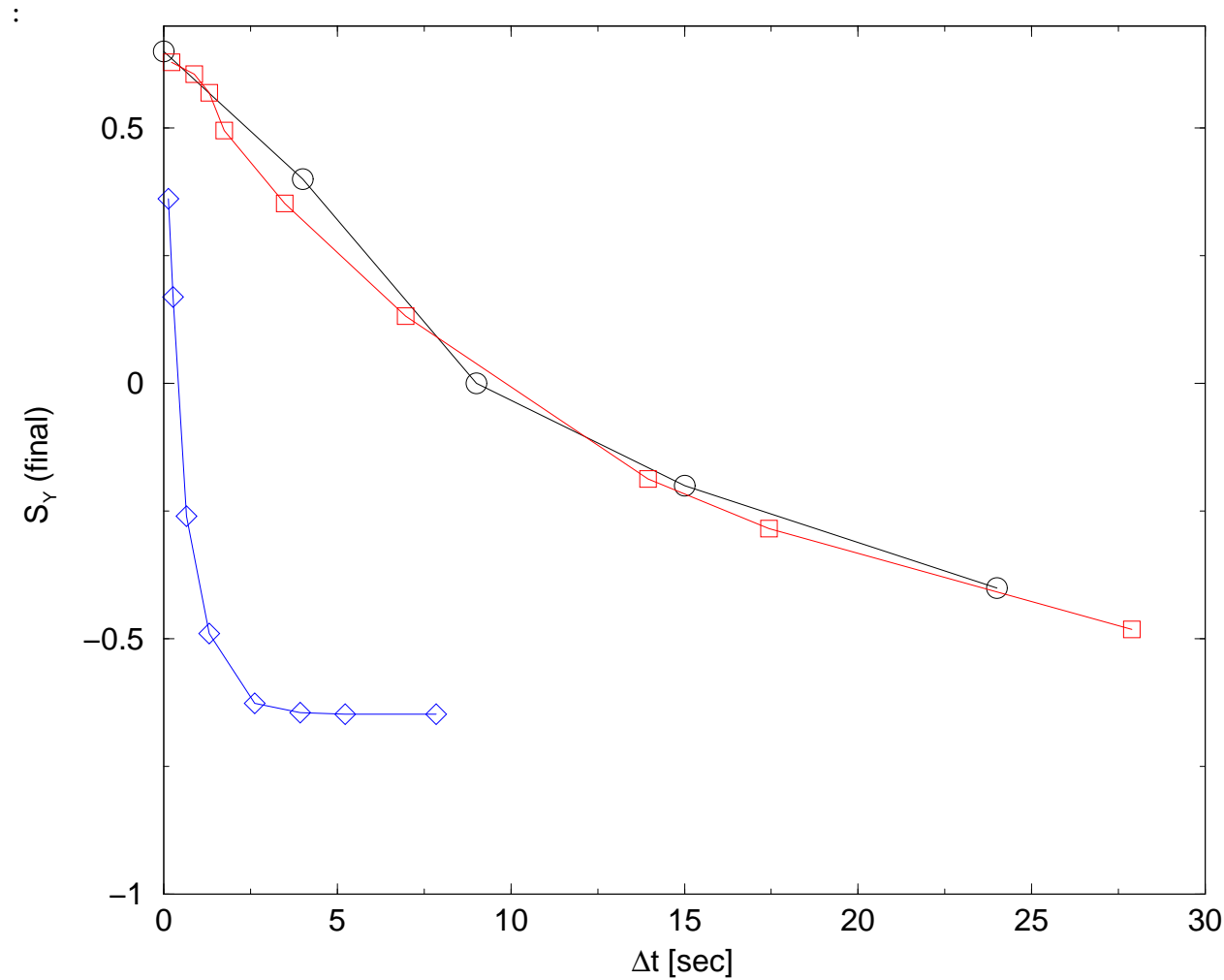

Figure 4: Comparison between Spink simulation and measured values for polarized deuterons resonanne induced by a RF Dipole. Circles indicate measured values for vector polarization, squares are values simulated with a $G \gamma$ coefficient in the RF Dipole, diamonds with $1+G \gamma$. The vertical axis is the vertical component of the spin, the horizontal axis is the duration of the RFD action, inversely proportional to the speed of resonance crossing. 


\section{Acknowledgments}

Discussions with E.D.Courant, Thomas Roser, Waldo MacKay, Vahid Ranjbar have been very important to write this note. The data for the COSY experiments were exposed to my attention during a recent visit to the Jülich Research Center by A.Lehrach with whom we are collaborating to extend the analysis to other data measured by the SPIN@COSY collaboration.

\section{References}

[1] A.U.Luccio: Spin Tracking in RHIC-Code SPINK. In: Y.Onel, N.PAVER, A.Penzo, editor: Proc. Adriatico Research Conf on Trends in Collider Spin Physics. Trieste, Italy, 12/5-8, 1995. World Scientific, p.235.

[2] A.U.Luccio: Spin Rotation Matrices for Spin Tracking. Technical Report BNL-62371, Brookhaven National Laboratory. Upton, NY, Oct.30 1995.

[3] S.Y.LeE: Spin Dynamics and Snakes in Synchrotrons. World Scientific. Singapore, 1997.

[4] E.D.Courant and R.D.Ruth: The Acceleration of Polarized Protons in Circular Accelerators. Technical Report BNL 51270, Brookhaven National Laboratory. Upton, NY, September 12, 1980.

[5] E.D.Courant: Revised Spin Motion Equations. Technical Report C-A/AP/292, Brookhaven National Laboratory. Upton, NY, Upton, NY.

[6] H.Grote and F.Ch.Iselin: The MAD program, Vers.8.19. Technical Report CERN/SL/90-13, European Organization for Nuclear Research, Geneva, CH, 1996.

[7] A.D.Krisch, M.A.Leonova, V.S.Morozov R.S.Raymond.D.W.Sivers V.K.Wong R.Gebel A Lehrach B.Lorentz R.Maier D.Prasuhn H.Stockhorst F.Hinterberger and K.UlbRICH: Unexpected reduction of rf spin resonance strength for stored deuteron beams. Phys.Rev.Spec.Topics-Accel.and Beams, (10):07100-1, 2007. 